\title{
A NEW KANTOROVICH-TYPE THEOREM FOR NEWTON'S METHOD
}

Abstract. A new Kantorovich-type convergence theorem for Newton's method is established for approximating a locally unique solution of an equation $F(x)=0$ defined on a Banach space. It is assumed that the operator $F$ is twice Fréchet differentiable, and that $F^{\prime}, F^{\prime \prime}$ satisfy Lipschitz conditions. Our convergence condition differs from earlier ones and therefore it has theoretical and practical value.

I. Introduction. In this study we are concerned with the problem of approximating a locally unique solution $x^{*}$ of the equation

$$
F(x)=0
$$

where $F$ is a twice Fréchet differentiable operator defined on a convex subset $D$ of a Banach space $E_{1}$ with values in a Banach space $E_{2}$.

Newton's method

$$
x_{n+1}=x_{n}-F^{\prime}\left(x_{n}\right)^{-1} F\left(x_{n}\right) \quad(n \geq 0), \quad x_{0} \in D,
$$

has been used extensively by many authors (see [1]-[6] and the references there) to generate a sequence $\left\{x_{n}\right\}_{n \geq 0}$ converging to $x^{*}$. In particular the following conditions have been used:

Condition A (Kantorovich [6]). Let $F: D \subseteq E_{1} \rightarrow E_{2}$ be Fréchet differentiable in $D, F^{\prime}\left(x_{0}\right)^{-1} \in L\left(E_{2}, E_{1}\right)$ for some $x_{0} \in D$, where $L\left(E_{2}, E_{1}\right)$ is the set of bounded linear operators from $E_{2}$ into $E_{1}$, and assume

$$
\begin{gathered}
\left\|F^{\prime}\left(x_{0}\right)^{-1}\left[F^{\prime}(x)-F^{\prime}(y)\right]\right\| \leq l\|x-y\| \quad \text { for all } x, y \in D \\
\left\|F^{\prime}\left(x_{0}\right)^{-1} F\left(x_{0}\right)\right\| \leq a
\end{gathered}
$$

1991 Mathematics Subject Classification: 65J15, 47H15, 49M17.

Key words and phrases: Newton's method, Banach space, Kantorovich hypothesis, Lipschitz-Hölder condition. 
and

$$
2 a l \leq 1 .
$$

Under condition A, one can obtain error estimates, existence and uniqueness regions of solutions, and know whether $x_{0}$ is a convergent initial guess, i.e., Newton's method (2) starting from $x_{0}$ converges to $x^{*}$. But sometimes when we want to determine whether the Newton iteration (2) starting from $x_{0}$ converges, Condition A fails.

ExAmple 1.1. Let $E_{1}=E_{2}=\mathbb{R}, D=[\sqrt{2}-1, \sqrt{2}+1], x_{0}=\sqrt{2}$ and define the real polynomial $F$ on $D$ by

$$
F(x)=\frac{1}{6} x^{3}-\alpha, \quad \alpha=\frac{2^{3 / 2}}{6}+.23 .
$$

Using (3), (4), (6) and the above choices we get $a=.23$ and $l=2.4142136$. Condition (5) is not satisfied since

$$
2 a l=1.1105383>1 .
$$

Therefore under condition A we cannot determine whether Newton's method (2) starting from $x_{0}=\sqrt{2}$ converges.

That is why in this study we introduce a new condition and a new theorem under which we will see that Newton's method starting from $x_{0}=\sqrt{2}$ in Example 1.1 converges.

From now on we assume:

Condition B. Let $F: D \subseteq E_{1} \rightarrow E_{2}$ be twice Fréchet differentiable in $D$, with $F^{\prime}(x) \in L\left(E_{1}, E_{2}\right), F^{\prime \prime}(x) \in L\left(E_{1}, L\left(E_{1}, E_{2}\right)\right)(x \in D), F^{\prime}\left(x_{0}\right)^{-1}$ exists at some $x_{0} \in D$, and assume

$$
\begin{aligned}
0<\left\|F^{\prime}\left(x_{0}\right)^{-1} F\left(x_{0}\right)\right\| \leq a, \quad\left\|F^{\prime}\left(x_{0}\right)^{-1} F^{\prime \prime}\left(x_{0}\right)\right\| \leq b, \\
\left\|F^{\prime}\left(x_{0}\right)^{-1}\left[F^{\prime}(x)-F^{\prime}\left(x_{0}\right)\right]\right\| \leq c\left\|x-x_{0}\right\|, \quad c>0, \\
\left\|F^{\prime}\left(x_{0}\right)^{-1}\left[F^{\prime \prime}(x)-F^{\prime \prime}\left(x_{0}\right)\right]\right\| \leq d\left\|x-x_{0}\right\| \quad \text { for all } x \in D,
\end{aligned}
$$

and

$$
2 k a \leq 1,
$$

where either

$$
k=\max \{c, b+2 a d\},
$$

or, if the function

$$
f(t)=t^{3}-2 b t^{2}-\left(2 d-b^{2}\right) t+2 d(b+a d)
$$

has two positive zeros $k^{1}, k^{2}$ such that

$$
[b, b+2 a d] \subseteq\left[k^{1}, k^{2}\right],
$$


then $k \geq c$ and

$$
k \in[b, b+2 a d]
$$

2. Convergence analysis. We need the lemma:

Lemma 2.1. Let $a, k$ be given positive constants. Define the real polynomial $p$ on $[0, \infty)$ by

$$
p(t)=\frac{k}{2} t^{2}-t+a
$$

and the iteration $\left\{t_{n}\right\}_{n \geq 0}$ by

$$
\begin{aligned}
t_{0} & =0, \\
t_{n+1} & =t_{n}-\frac{p\left(t_{n}\right)}{p^{\prime}\left(t_{n}\right)} .
\end{aligned}
$$

Assume

$$
2 k a \leq 1
$$

Then the equation

$$
p(t)=0
$$

has two positive roots $r_{1}, r_{2}$ with $r_{1} \leq r_{2}$ and the iteration $\left\{t_{n}\right\}_{n \geq 0}$ generated by (16)-(17) is such that $t_{0}<t_{1}<\ldots<t_{n}<t_{n+1}<\ldots<r_{1}$ with $\lim _{n \rightarrow \infty} t_{n}=r_{1}$.

Proof. Using (15) and (18) we deduce that equation $p(t)=0$ has two positive roots

$$
r_{1}=\frac{1-\sqrt{1-2 k a}}{k} \text { and } r_{2}=\frac{1+\sqrt{1-2 k a}}{k}
$$

with $r_{1} \leq r_{2}$. Moreover the function $t-p(t) / p^{\prime}(t)$ increases on $\left[0, r_{1}\right]$, since $p^{\prime}(t)<0, p^{\prime \prime}(t)>0$ and $p(t)>0$ on $\left[0, r_{1}\right]$. Furthermore if $t_{n} \in\left[0, r_{1}\right]$ for all integer values smaller than or equal to $n$, then we obtain

$$
t_{n} \leq t_{n}-\frac{p\left(t_{n}\right)}{p^{\prime}\left(t_{n}\right)}=t_{n+1} \quad \text { and } \quad t_{n+1}=t_{n}-\frac{p\left(t_{n}\right)}{p^{\prime}\left(t_{n}\right)} \leq r_{1}-\frac{p\left(r_{1}\right)}{p^{\prime}\left(r_{1}\right)}=r_{1} \text {. }
$$

We set $\bar{U}\left(x_{0}, s\right)=\left\{x \in E_{2} \mid\left\|x-x_{0}\right\| \leq s\right\}$ and $U\left(x_{0}, s\right)=\left\{x \in E_{1} \mid\right.$ $\left.\left\|x-x_{0}\right\|<s\right\}$.

Lemma 2.2. The following estimates are true for $x \in U\left(x_{0}, 1 / c\right)$ :

$$
\left\|F^{\prime}(x)^{-1} F^{\prime}\left(x_{0}\right)\right\| \leq\left(1-c\left\|x-x_{0}\right\|\right)^{-1}
$$

and

$$
\left\|F^{\prime}\left(x_{0}\right)^{-1} F^{\prime \prime}(x)\right\| \leq b+d\left\|x-x_{0}\right\| .
$$


Pr o of. If $x \in U\left(x_{0}, 1 / c\right)$, using (7), the estimate

$$
\left\|F^{\prime}\left(x_{0}\right)^{-1}\left(F^{\prime}(x)-F^{\prime}\left(x_{0}\right)\right)\right\| \leq c\left\|x-x_{0}\right\|<1,
$$

and the Banach lemma on invertible operators [6], the operator $F^{\prime}(x)$ has a continuous inverse on $U\left(x_{0}, 1 / c\right)$ and

$$
\left\|F^{\prime}(x)^{-1} F^{\prime}\left(x_{0}\right)\right\| \leq\left(1-c\left\|x-x_{0}\right\|\right)^{-1} .
$$

Moreover by (6) and (11) we get

$$
\begin{aligned}
\left\|F^{\prime}\left(x_{0}\right)^{-1} F^{\prime \prime}(x)\right\| & \leq\left\|F^{\prime}\left(x_{0}\right)^{-1} F^{\prime \prime}\left(x_{0}\right)\right\|+\left\|F^{\prime}\left(x_{0}\right)^{-1}\left(F^{\prime \prime}(x)-F^{\prime \prime}\left(x_{0}\right)\right)\right\| \\
& \leq b+d\left\|x-x_{0}\right\| .
\end{aligned}
$$

We can now prove the following semilocal result concerning the convergence of Newton's method (2).

Theorem 2.3. Let $F$ be the operator defined in (1). Let $p$ be the polynomial defined in (15). Assume that $U\left(x_{0}, 1 / c\right) \subseteq D$ and Condition $B$ holds. Then Newton's iteration $\left\{x_{n}\right\}_{n \geq 0}$ generated by (2) is well defined, remains in $\bar{U}\left(x_{0}, r_{1}\right)$ for all $n \geq 0$, and converges to a solution $x^{*} \in \bar{U}\left(x_{0}, r_{1}\right)$ of the equation $F(x)=0$, which is unique in $U\left(x_{0}, r_{2}\right)$ if $r_{1}<r_{2}$. If $r_{1}=r_{2}$ the solution $x^{*}$ is unique in $\bar{U}\left(x_{0}, r_{1}\right)$. Moreover the following estimates hold for all $n \geq 0$ :

$$
\left\|x_{n+1}-x_{n}\right\| \leq t_{n+1}-t_{n}
$$

and

$$
\left\|x_{n}-x^{*}\right\| \leq r_{1}-t_{n}=\left(r_{1} / r_{2}\right)^{2^{n}}\left(r_{2}-t_{n}\right)
$$

where $r_{1}$ and $r_{2}$ are the roots of the quadratic equation $p(t)=0$ given by (20).

Proof. Using induction on $n$ we first show estimate (23). The approximation $x_{1}$ is defined and

$$
\left\|x_{1}-x_{0}\right\|=\left\|F^{\prime}\left(x_{0}\right)^{-1} F\left(x_{0}\right)\right\| \leq a=t_{1}-t_{0}<r_{1} .
$$

It follows that $x_{1} \in \bar{U}\left(x_{0}, r_{1}\right)$ and (23) holds for $n=0$.

Assume that (23) holds for all integer values $i \leq n$. Using (2) we can write in turn

$$
\begin{aligned}
F^{\prime}\left(x_{0}\right)^{-1} F\left(x_{i+1}\right)= & F^{\prime}\left(x_{0}\right)^{-1}\left[F\left(x_{i+1}\right)-F\left(x_{i}\right)-F^{\prime}\left(x_{i}\right)\left(x_{i+1}-x_{i}\right)\right] \\
= & F^{\prime}\left(x_{0}\right)^{-1}\left\{\int _ { 0 } ^ { 1 } \left[F^{\prime \prime}\left[x_{i}+t\left(x_{i+1}-x_{i}\right)\right]\right.\right. \\
& \left.-F^{\prime \prime}\left(x_{0}\right)\right](1-t) d t\left(x_{i+1}-x_{i}\right)^{2} \\
& \left.+\frac{1}{2} F^{\prime \prime}\left(x_{0}\right)\left(x_{i+1}-x_{i}\right)^{2}\right\}
\end{aligned}
$$


Using the induction hypothesis we have

$$
\left\|x_{i+1}-x_{0}\right\| \leq \sum_{j=1}^{i+1}\left\|x_{j}-x_{j-1}\right\| \leq \sum_{j=1}^{i+1}\left(t_{i}-t_{i-1}\right)=t_{i+1}-t_{0}=t_{i+1}<r_{1}
$$

and

$$
\left\|x_{i}+t\left(x_{i+1}-x_{i}\right)-x_{0}\right\| \leq t_{i}+t\left(t_{i+1}-t_{i}\right)<r_{1} .
$$

Hence, by (7), (9), (15), (22), (23) and (25) we get

$$
\begin{aligned}
\| F^{\prime}\left(x_{0}\right)^{-1} F( & \left.x_{i+1}\right) \| \\
& \leq \frac{1}{2}\left[b+d\left\|x_{i}-x_{0}\right\|+\frac{d}{3}\left\|x_{i+1}-x_{i}\right\|\right]\left\|x_{i+1}-x_{i}\right\|^{2} \\
& \leq \frac{1}{2}\left[b+d t_{i}+\frac{d}{3}\left(t_{i+1}-t_{i}\right)\right]\left(t_{i+1}-t_{i}\right)^{2} \\
& \leq \frac{1}{2}\left[b+\frac{2}{3} d t_{i}+\frac{d t_{i+1}}{3}\right]\left(t_{i+1}-t_{i}\right)^{2} \\
& \leq \frac{1}{2}\left[b+\frac{2}{3} d r_{1}+\frac{d r_{1}}{3}\right]\left(t_{i+1}-t_{i}\right)^{2} \\
& \leq \frac{k}{2}\left(t_{i+1}-t_{i}\right)^{2} \leq p\left(t_{i+1}\right) .
\end{aligned}
$$

By (2), (17), (21) and (26) we obtain

$$
\left\|x_{i+2}-x_{i+1}\right\| \leq-\frac{p\left(t_{i+1}\right)}{p^{\prime}\left(t_{i+1}\right)}=t_{i+2}-t_{i+1},
$$

which shows (23) for all $n \geq 0$.

By Lemma 2.1 and estimate (23) it follows that $\left\{x_{n}\right\}_{n \geq 0}$ is a Cauchy sequence in the Banach space $E_{1}$ and so it converges to some limit $x^{*} \in$ $\bar{U}\left(x_{0}, r_{1}\right)$ (since $\bar{U}\left(x_{0}, r_{1}\right)$ is a closed set). By (2) and the continuity of $F$, we get $F\left(x^{*}\right)=0$. To show uniqueness let $y \in U\left(x_{0}, r_{2}\right)$ be such that $F(y)=0$. Using (2) we obtain

$$
\begin{aligned}
y-x_{n+1}= & -\left[F^{\prime}\left(x_{n}\right)^{-1} F^{\prime}\left(x_{0}\right)\right]\left\{\int _ { 0 } ^ { 1 } F ^ { \prime } ( x _ { 0 } ) ^ { - 1 } \left(F^{\prime \prime}\left(x_{n}+t\left(y-x_{n}\right)\right)\right.\right. \\
& \left.-F^{\prime \prime}\left(x_{0}\right)\right)(1-t) d t\left(y-x_{n}\right)^{2} \\
& \left.+\int_{0}^{1} F^{\prime}\left(x_{0}\right)^{-1} F^{\prime \prime}\left(x_{0}\right)(1-t) d t\left(y-x_{n}\right)^{2}\right\} .
\end{aligned}
$$

As in (25), (26) we get $\left\|y-x_{0}\right\| \leq r_{1}-t_{0}$ if $y \in \bar{U}\left(x_{0}, r_{1}\right)$, and $\left\|y-x_{0}\right\|=$ $\lambda\left(r_{2}-t_{0}\right), 0<\lambda<1$, if $y \in U\left(x_{0}, r_{2}\right)$. That is, as in (25), by (27) we have $\left\|y-x_{n}\right\| \leq r_{1}-t_{n}$ if $y \in \bar{U}\left(x_{0}, r_{1}\right)(n \geq 0)$, and $\left\|y-x_{n}\right\| \leq \lambda^{2^{n}}\left(r_{2}-t_{n}\right)$ if 
$y \in U\left(x_{0}, r_{2}\right)(n \geq 0)$. From the above estimates and $F\left(x^{*}\right)=0$ it follows that $x^{*}=\lim _{n \rightarrow \infty} x_{n}=y$ in either case.

Finally estimates (24) follow by using standard majorization techniques, (17) and (23) ([2], [3], [6]).

\section{Applications and concluding remarks}

Remark 3.1. Let us apply Theorem 2.3 to Example 1.1. By (7)-(9), (11) we get $a=.23, b=\sqrt{2}, c=\sqrt{2}+.5, d=1$, and $k=1.9142136$. Then condition (10) becomes

$$
2 k a=.8805383<1,
$$

which is true. Hence equation (6) has a solution $x^{*} \in U(\sqrt{2}, 1)$. Moreover Newton's method (2) starting from $x_{0}=\sqrt{2}$ converges quadratically to $x^{*}$. We also remark that as we noted in Example 1.1, Condition A fails to determine whether Newton's method converges in this case. We found $x^{*}=1.614507$.

REMARK 3.2. The convergence of Newton's method (2) can be established independently using Conditions A and B. In practice we can use both of them to determine the smallest region where the solution is located and the largest one where this solution is unique. Let us make such a comparison between Conditions A and B. Consider the polynomial $q$ given by

$$
q(t)=\frac{l}{2} t^{2}-t+a
$$

with roots denoted by $r_{3}$ and $r_{4}\left(r_{3} \leq r_{4}\right)$. Then since $c \leq l$ we find from (15) that $p\left(r_{3}\right) \leq 0$ and $p\left(r_{4}\right) \leq 0$. Hence we get $r_{1} \leq r_{3} \leq r_{4} \leq r_{2}$ and $r_{3} \leq 1 / c$. Note also that our theorem uses simply a quadratic polynomial $p$ and condition (10) instead of a cubic polynomial and condition (27) in [3], [5] (which are more difficult to handle in general).

REMARK 3.3. We can extend the result obtained in Theorem 2.3 to include the Hölder case. Assume, instead of (9) in Condition B, that F satisfies

$$
\begin{aligned}
& \left\|F^{\prime}\left(x_{0}\right)^{-1}\left[F^{\prime \prime}(x)-F^{\prime \prime}\left(x_{0}\right)\right]\right\| \leq d_{0}\left\|x-x_{0}\right\|^{q} \\
& \quad \text { for all } x \in D, q \in[0,1] \text { and some } d_{0} \geq 0 .
\end{aligned}
$$

For $q=0$, we obtain $\left\|F^{\prime}\left(x_{0}\right)^{-1} F^{\prime \prime}(x)\right\| \leq d_{0}+b$, and we are in the situation of the Kantorovich theorem [6, Theorem XVIII.1.6]. If $q=1$ in (28) we get (9). Moreover if $q \in(0,1)$, then $F^{\prime \prime}$ is $q$-Hölder continuous on $D$. Let $a, b$, $c$ be as before. Assume there exists $k_{0} \geq c$ such that $b+d_{0} r_{1}^{q} \geq k_{0}$, where $r_{1}$ is given by (20), and condition (10) holds with $k$ replaced by $k_{0}$.

With the above changes the conclusions of Theorem 2.3 hold for the Hölder case. 


\section{References}

[1] I. K. Argyros, Newton-like methods under mild differentiability conditions with error analysis, Bull. Austral. Math. Soc. 37 (1988), 131-147.

[2] I. K. Argyros and F. Szidarovszky, The Theory and Applications of Iteration Methods, C.R.C. Press, Boca Raton, Fla., 1993.

[3] J. M. Gutiérrez, A new semilocal convergence theorem for Newton's method, J. Comput. Appl. Math. 79 (1997), 131-145.

[4] J. M. Gutiérrez, M. A. Hernández, and M. A. Salanova, Accessibility of solutions by Newton's method, Internat. J. Comput. Math. 57 (1995), 239-247.

[5] Z. Huang, A note on the Kantorovich theorem for Newton iteration, J. Comput. Appl. Math. 47 (1993), 211-217.

[6] L. V. Kantorovich and G. P. Akilov, Functional Analysis, Pergamon Press, Oxford, 1982.

Ioannis K. Argyros

Cameron University

Department of Mathematics

Lawton, Oklahoma 73505

U.S.A.

E-mail: Ioannisa@cameron.edu 